Review Article

\title{
Cell Transplantation for Spinal Cord Injury: Tumorigenicity of Induced Pluripotent Stem Cell-Derived Neural Stem/Progenitor Cells
}

\author{
Junhao Deng $(\mathbb{D}$, Yiling Zhang $\mathbb{D}$, Yong Xie $\mathbb{D}$, Licheng Zhang $\mathbb{D}$, and Peifu Tang $\mathbb{D}$ \\ Department of Orthopaedics, Chinese PLA General Hospital, No. 28 Fuxin Road, Beijing 100853, China \\ Correspondence should be addressed to Peifu Tang; pftang301@163.com
}

Received 16 July 2017; Accepted 21 December 2017; Published 4 February 2018

Academic Editor: Ming Su

Copyright ( $\odot 2018$ Junhao Deng et al. This is an open access article distributed under the Creative Commons Attribution License, which permits unrestricted use, distribution, and reproduction in any medium, provided the original work is properly cited.

\begin{abstract}
Spinal cord injury (SCI) is an intractable and worldwide difficult medical challenge with limited treatments. Neural stem/progenitor cell (NS/PC) transplantation derived from fetal tissues or embryonic stem cells (ESCs) has demonstrated therapeutic effects via replacement of lost neurons and severed axons and creation of permissive microenvironment to promote repair of spinal cord and axon regeneration but causes ethnical concerns and immunological rejections as well. Thus, the implementation of induced pluripotent stem cells (iPSCs), which can be generated from adult somatic cells and differentiated into NS/PCs, provides an effective alternation in the treatment of SCI. However, as researches further deepen, there is accumulating evidence that the use of iPSC-derived NS/PCs shows mounting concerns of safety, especially the tumorigenicity. This review discusses the tumorigenicity of iPSC-derived NS/PCs focusing on the two different routes of tumorigenicity (teratomas and true tumors) and underlying mechanisms behind them, as well as possible solutions to circumvent them.
\end{abstract}

\section{Background}

Spinal cord injury is a devastating neurological condition, which results in the disruption of signals between the brain and body yielding severe physical, psychological, and social dysfunction $[1,2]$. Patients who have suffered a SCI not only become increasingly dependent on others for daily life but are more likely to die prematurely and are at risk for social exclusion $[1,2]$. What is worse is that, due to the complex pathophysiological processes, significant treatment for SCI has progressed slowly.

Originally, glucocorticoid drugs like methylprednisolone were regarded as the classic therapeutic treatment for SCI [3], as they had been found to stabilize the plasma membrane of damaged cells by inhibiting lipid peroxidation and hydrolysis [3]. However, their application gradually became controversial because they had serious side effects like mounting vulnerability to acute corticosteroid myopathy or serious infection $[4,5]$. Other clinical approaches to SCI included early surgical interventions [6] and alternative pharmacological therapy (e.g., GM-1 [7] and thyrotropin- releasing hormone [8]). However, these methods either had their own side effects or demonstrated weakly therapeutic efficacy.

Recent progress in cell transplantation has opened up new opportunities to understand and treat SCI. Among the several types of candidate cells, NS/PC holds great therapeutic potential for SCI, as it can replace the lost neurons and glia as well as create a growth-promoting environment [9]. Nevertheless, the acquisition of NS/PCs can be a difficult task since they are usually located deep in the brain so their isolation is a highly invasive procedure. To bypass this problem, people have also used ESCs from which they can generate sufficient NS/PCs. Indeed, ESC-derived NS/PCs were initially reported to have optimistic effects on SCI $[10,11]$. Unfortunately, the application of ESC-based strategy, accompanied by immune rejections and ethical concerns [12], was less likely to be transformed into clinical practice. Subsequently, the advent of iPSCs appears to signal the future of stem cell treatments for SCI. However, while the therapeutic effects of iPSCs on SCI have been discussed by many studies, the side effects are rarely mentioned and talked over exclusively, 
especially the tumorigenicity of iPSCs. In this paper, we briefly summarized the application of iPSCs, elucidated the tumorigenicity in detail, and described possible strategies to address it.

\section{Application of iPSCs in Spinal Cord Injury: An Overview}

In 2006, Takahashi and Yamanaka showed that fibroblasts from mouse somatic cells could regain pluripotency after expressing four transcriptional factors [13], thus developing iPSCs. It stands to reason that iPSCs may have the greatest potential for regenerative medicine, because they have abilities to indefinitely self-renew and differentiate into most if not all cell types $[13,14]$. Compared to ESCs, autologous iPSCs also circumvent the ethical issues associated with embryonic tissue harvesting and free patients of immunosuppression, which is critical since SCI patients are at high risk for infection [15].

Of late, an increasing number of research groups have applied iPSCs to SCI and achieved interesting results (Table 1). In 2010, Tsuji et al. managed to produce neurospheres from mouse iPSCs and showed that transplantation of these cells promoted functional improvement in mice with SCI [16]. As a proof of principle, they also used human iPSCs (hiPSCs) and demonstrated significant therapeutic effects like the better recovery of motor function, synapse formation between the grafts and hosts, and enhanced axonal regrowth [17]. Kobayashi et al. transplanted hiPSC-derived NS/PCs into a nonhuman primate following cervical SCI and revealed behavioral improvements consistent with rodent studies [18]. Lu et al. reported that not only can the derivatives of iPSCs extend axons over nearly the whole length of the rat CNS [19] but can also form extensive synaptic connections with the host. More recently, several studies have elucidated potential mechanisms underlying behavioral improvement from SCI following transplantation of iPSC derivatives [20, 21]. They speculated that iPSC derivatives exerted their effects on SCI by substitution of lost neural cells, promotion of axonal remyelination, and regrowth as well as tissue sparing through trophic support.

There are also some negative reports on iPSC approaches to SCI. Two reports revealed that despite the ability to differentiate into neural cells [19, 22], iPSCderived NS/PCs did not show any substantial improvement in function. Besides, it takes a long time to generate and evaluate iPSCs [23], making it unrealistic for individualized iPSC-based therapy because the optimal time for stem cell transplantation is the subacute phase [24]. As a result, either iPSCs would have to be generated from donor tissue, missing out on the major factor that makes them attractive in the first place, or transplanted at more chronic phases of injury [25], which showed a poor result after transplantation into the chronic SCI model. More importantly, like ESCs, there are widely found issues with respect to safety of iPSCs, particularly the possible tumorigenicity $[16,21,26]$.

\section{Characteristics and Underlying Mechanisms of iPSC in Tumorigenicity}

Tumorigenicity of any stem cell transplants remains a major concern for clinical applications, and there is an urgent need for it to be addressed before translation of iPSC techniques into SCI treatment. From several reports [26, 27], tumorigenicity of iPSCs can be classified into two distinct types: teratoma and true tumors due to their different features and developmental processes, which we will discuss further below (Figure 1).

3.1. Teratoma Formation. Teratoma is a relatively common potential risk in grafts of iPSCs especially when individual iPSC clones were preevaluated as unsafe $[16,17,28]$. While the mechanism is not fully understood, most reports share the idea that undifferentiated iPSCs lead to teratoma formation $[26,29]$. Teratoma formation requires the ability to escape or silence the immune responses for the purpose of survival in the host. Tumor cells could take effective measures to avoid immune responses by alteration of MHC-I, mutations in Fas or Trail, and so forth [30]. These traits are well shared with undifferentiated iPSCs. Besides, like tumor cells, iPSCs possess a virtually unlimited proliferation potential, by which they are vulnerable to the formation of a cell mass. Therefore, we reasonably postulate that residualundifferentiated cells contribute greatly to teratoma formation. Moreover, Miura et al. discovered that the presence or absence of c-Myc in iPSCs and drug selection for NANOG or Fbxo15 expression [28, 31], all of which are considered closely associated with tumorigenesis, showed no correlation with teratoma formation. Namely, the underlying mechanism of teratoma formation is different from that of tumor, as they do not correlate with these tumor makers.

It is still unclear why undifferentiated cells remain in iPSC grafts. However, iPSC derivatives of different origins do demonstrate different teratoma-forming propensity $[16,28]$. For instance, iPSCs derived from tail-tip fibroblasts showed the highest propensity for teratoma formation while iPSCs from embryonic fibroblasts and gastric epithelial cells showed the lowest. Since iPSCs from different origins exhibited distinctive features, it is possible that epigenetic memory, the residual features of somatic tissues, plays a role in teratoma formation. And due to epigenetic memory, iPSCs from certain cell lines may be likely to redifferentiate back into their initial cell type $[32,33]$. Therefore, we might as well hold the belief that if we created a certain type of microenvironment supporting certain iPSCs to differentiate into NS/ PCs, those derived from any other cell lines except neural ones may not be able to well differentiate and have to maintain undifferentiated status under this unfavorable condition. Besides, the inefficient methods of purifying the contaminated undifferentiated cells also aggravate the situation.

3.2. Substantial Tumorigenesis. Several studies have found that even if all undifferentiated cells are purged [26, 34], iPSC derivatives remain tumorigenic, as substantial tumors were present instead of teratomas. Such cases can be much worse because they are usually malignant and able to progress, 
TABLE 1: Some current studies of iPS cell transplantation in the SCI model. This table summarizes some of the experimental studies involving iPSC derivative transplantation into SCI models and its outcomes.

\begin{tabular}{|c|c|c|c|}
\hline Reference & Grafts & SCI model & Outcomes \\
\hline $\begin{array}{l}\text { Tsuji et al. } \\
{[16]}\end{array}$ & Mouse iPSC-derived neurospheres & $\begin{array}{l}\text { Mouse contusive SCI } \\
\text { at T-10 level }\end{array}$ & $\begin{array}{l}\text { Preevaluated safe iPSC-derived cells promoted functional } \\
\text { recovery without teratomas or other tumors, while the } \\
\text { "unsafe" iPSC-derived cell resulted in teratoma forming and } \\
\text { functional deterioration }\end{array}$ \\
\hline $\begin{array}{l}\text { Nori et al. } \\
{[17]}\end{array}$ & Human iPSC-derived neurospheres & $\begin{array}{l}\text { Mouse contusive SCI } \\
\text { at T-10 level }\end{array}$ & $\begin{array}{l}\text { Human iPSC-derived cells improved motor functional } \\
\text { recovery without tumor formation but some pluripotent } \\
\text { stem cells remained at } 112 \mathrm{~d} \text { post-SCI }\end{array}$ \\
\hline $\begin{array}{l}\text { Kobayashi } \\
\text { et al. [18] }\end{array}$ & Human iPSC-derived NS/PCs & $\begin{array}{l}\text { Marmoset contusive } \\
\text { SCI at C-5 level }\end{array}$ & $\begin{array}{l}\text { Preevaluated safe iPSC-derived cells promoted functional } \\
\text { recovery without tumors, whereas some undifferentiated } \\
\text { cells were still presented after } 16 \text { weeks posttransplantation }\end{array}$ \\
\hline $\begin{array}{l}\text { Fujimoto } \\
\text { et al. [20] }\end{array}$ & $\begin{array}{l}\text { Human iPSC-derived neuroepithelial- } \\
\text { like stem cells (hiPS-It-NESC) }\end{array}$ & $\begin{array}{l}\text { Mouse contusive SCI } \\
\text { at T9-10 level }\end{array}$ & $\begin{array}{l}\text { hiPS-It-NESCs promote recovery of motor function and } \\
\text { reconstruct neuronal circuity with no tumors up to } 12 \\
\text { weeks after SCI }\end{array}$ \\
\hline $\begin{array}{l}\text { LiuTang } \\
\text { et al. } 2013\end{array}$ & Human iPSC-derived NSCs & $\begin{array}{c}\text { Rhesus monkey } \\
\text { contusive SCI at T9 } \\
\text { level }\end{array}$ & $\begin{array}{l}\text { Human iPSC-derived NSCs migrated into damaged regions } \\
\text { and showed functional recovery with no tumors after } 30 \\
\text { days post-SCI }\end{array}$ \\
\hline Lu et al. [19] & Human iPSC-NSCs & $\begin{array}{l}\text { Rats and mice lateral } \\
\text { spinal cord lesions at } \\
\text { C5 level }\end{array}$ & $\begin{array}{l}\text { Human iPSC-NSCs showed long-distance growth of human } \\
\text { axons without obvious functional recovery }\end{array}$ \\
\hline $\begin{array}{l}\text { Salewski } \\
\text { et al. } 2015\end{array}$ & Mouse iPSC-NSCs & $\begin{array}{l}\text { Clip-compression } \\
\text { spinal cord injuries at } \\
\text { the T6 level }\end{array}$ & $\begin{array}{l}\text { Wildtype-iPSC-NSCs improved neurobehavioral function } \\
\text { while nonmyelinating Shiverer-iPSC-NSC did not }\end{array}$ \\
\hline $\begin{array}{l}\text { Oh et al. } \\
2015\end{array}$ & iPSC-NPCs from human disc & $\begin{array}{l}\text { Mouse compressional } \\
\text { SCI at T-11 level }\end{array}$ & $\begin{array}{l}\text { iPSC-NPCs promoted functional and structural recovery } \\
\text { with no tumor formation but undifferentiated cells still } \\
\text { existed five weeks later }\end{array}$ \\
\hline $\begin{array}{l}\text { Pomeshchik } \\
\text { et al. [22] }\end{array}$ & Human iPSC-NPCs & $\begin{array}{l}\text { Mouse contusive SCI } \\
\text { at T-10 level }\end{array}$ & $\begin{array}{l}\text { Transplanted cells failed to improve functional recovery but } \\
\text { no tumor formed and undifferentiated cells were not } \\
\text { detected }\end{array}$ \\
\hline $\begin{array}{l}\text { Kawabata } \\
\text { et al. } 2016\end{array}$ & Human iPSC-OPC-enriched NS/PCs & $\begin{array}{l}\text { Mouse contusive SCI } \\
\text { at T-10 level }\end{array}$ & $\begin{array}{l}\text { Transplanted cells lead to robust remyelination and } \\
\text { enhance functional recovery without tumorigenicity }\end{array}$ \\
\hline $\begin{array}{l}\text { Okubo et al } \\
{[21]}\end{array}$ & $\begin{array}{l}\text { Human iPSC-NS/PCs with } \gamma \text {-secretase } \\
\text { inhibitor (GSI) }\end{array}$ & $\begin{array}{l}\text { Mouse contusive SCI } \\
\text { at T-10 level }\end{array}$ & $\begin{array}{l}\text { GSI-treated hiPSC-NS/PCs exhibited motor functional } \\
\text { recovery and decreased residual immature cells }\end{array}$ \\
\hline $\begin{array}{l}\text { Itakura et al } \\
\text { [27] }\end{array}$ & $\begin{array}{l}\text { Human-integrated iCaspase9-iPSC- } \\
\text { NS/PCs with chemical inducers of } \\
\text { dimerization (CIDs) }\end{array}$ & $\begin{array}{l}\text { Mouse contusive SCI } \\
\text { at T-10 level }\end{array}$ & $\begin{array}{l}\text { Transplanted cells of the CID group exhibited continually } \\
\text { functional recovery while the control groups showed } \\
\text { functional decline due to teratomas }\end{array}$ \\
\hline
\end{tabular}

invade, and metastasize. As such, understanding the mechanisms behind tumorigenesis is imperative.

The exact mechanism underlying iPSC tumorigenesis is still not clearly defined, but several factors are thought to contribute to it. Collectively, genomic and epigenomic instability correlates largely with tumorigenicity of iPSCs $[35,36]$. Many factors can account for genomic instability. For instance, several oncogenes (like c-Myc and KLF4) or genes sometimes associated with tumorigenesis (like SOX2 and Oct-4) are used in the reprogramming process. Additionally, retroviral or lentiviral gene delivery systems are used in the reprogramming process and can be integrated into the genome-disrupting tumor suppressor genes and pathways. For example, the activation of transgenic Oct-4 and KLF4 has been found to induce tumor formation of NS/PCs via the $\mathrm{Wnt} / \beta$-catenin signaling pathway [34]. This pathway was found to be able to enhance stabilization of telomeres, a signature of tumorigenesis, by increasing TERT expression. Furthermore, the mature cells harvested for iPSC induction have themselves already undergone multiple rounds of division and might possess their own genetic instability before induction [37]. Also, the low-efficiency reprogramming process and incomplete suppression of transgenic factors result in some partially reprogramming cells, which take part in tumor forming.

On the other hand, epigenomic instability, especially DNA methylation, also plays a role in the formation of true tumors [26]. DNA methylation has been found to have strong association with tumorigenesis in cancer tissues [38]. For instance, if oncogenes possess hypomethylation in a cell sample, such cells may show a higher likelihood to form tumors and vice versa. Consistent with this idea, 253G1hiPSCs as well as 253G1-iPSC-NS/PCs, which had DNA hypomethylation mainly in oncogenes and hypermethylation in tumor suppressor genes, were more likely to develop tumors when compared with 207B1-hiPSCs and NS/PCs, which did not. In addition, tumorigenicity can be enhanced as induced cells are passaged because the passage of iPSCs 


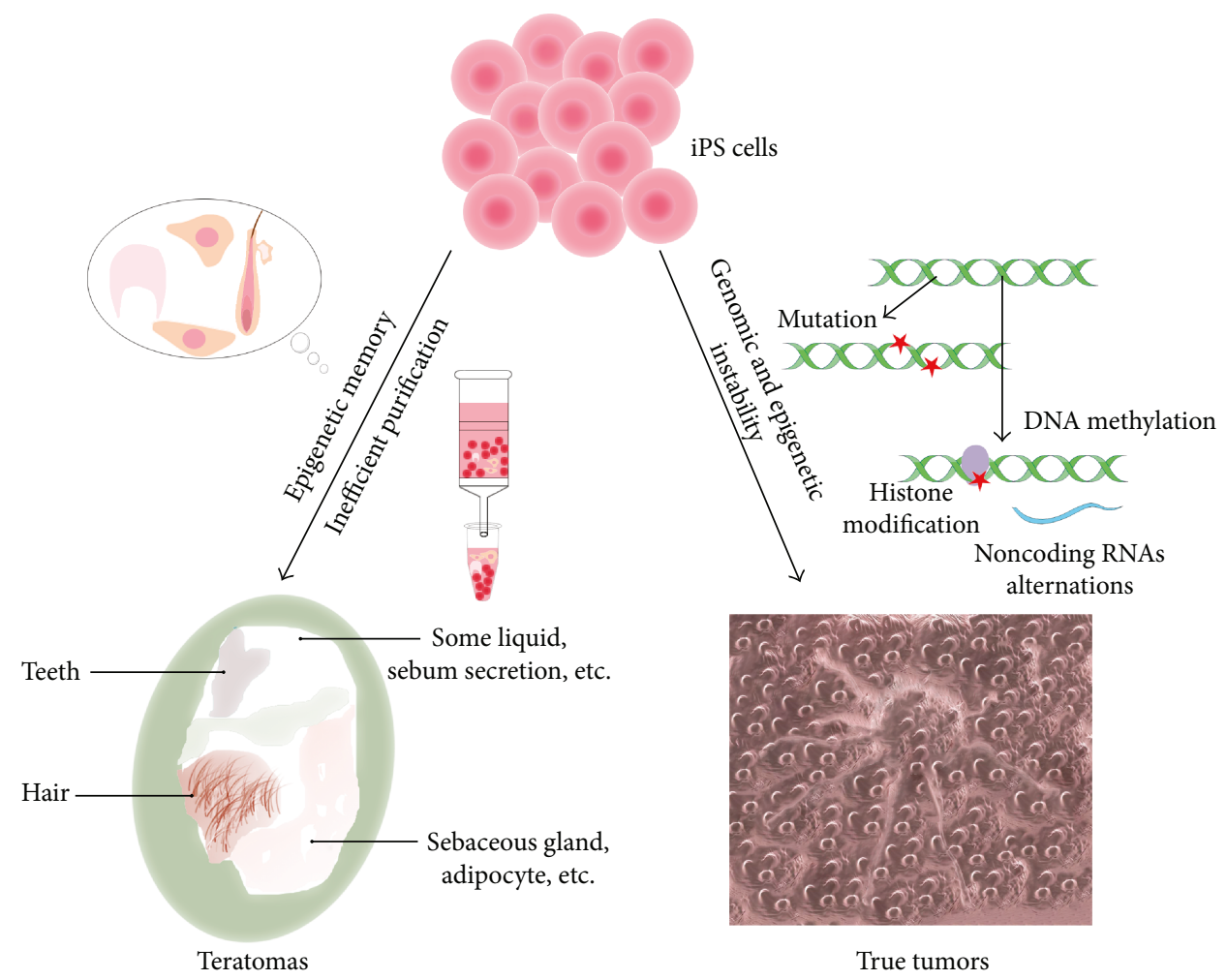

FIGURE 1: Two distinguished aspects of tumorigenicity and respective potential mechanisms. The tumorigenicity of iPSCs included two parts: one of them is teratoma formation and another is substantial tumor formation. The former is mostly attributed to epigenetic memory as well as inefficient purification, while the latter is ascribed to genomic instability and epigenetic changes.

and iPSC-derived NS/PCs further alters the epigenetic profiles via DNA methylation.

\section{Possible Solutions to iPSC Tumorigenesis}

4.1. Strategies to Prevent Teratoma Formation. As mentioned above, the formation of teratomas is largely attributed to undifferentiated cells. Based on this, some reports proposed various methods to address this problem including the following:

(1) Increased number of passages to weaken epigenetic memory. Several studies observed the loss of epigenetic memory with increased passage number $[33,39]$. iPSCs at late passage and ESCs became indistinguishable and acquired similar ability of differentiation. Therefore, the undifferentiated cell correspondingly reduced when iPSCs were capable enough of differentiation into other cells. While the underlying mechanism is not quite clear, two possible aspects may account for this phenomenon: (i) most of the iPSCs will gradually erase somatic marks as those cells passaged and/or (ii) those rare, fully reprogrammed cells become superior and then are picked up step by step [39].

(2) Take advantage of epigenetic memory characteristics and use it to reprogram iPSCs away from a teratoma-inducing lineage. The propensity of iPSCs to differentiate bias into their starting cell lineage could be exploited to produce certain cell types. For example, to get more NS/PCs from iPSCs, we may ideally think of the utilization of neural cells. Some previous reports $[40,41]$ also confirmed that, in comparison with other cell lineages of origin, iPSCs from neural tissue are more likely and efficient to differentiate into NS/PCs. The more likely to differentiate into other cells, the less possibility of forming teratomas.

(3) Improve the ability to purify iPSC-NS/PCs. It is essential to better gain bona fide iPSC-NS/PCs, as the potential for contamination with undifferentiated iPSCs presents a big chance of forming teratomas. Therefore, scientists have tried many ways to achieve the common goal including finding more specific cell surface makers and diminishing residual undifferentiated cells like inhibiting DNA topoisomerase II or stearoyl-coA desaturase [21, 42]. Accordingly, it does help but it still urgently needs to pan for desired unique makers or proper methods of depleting undifferentiated cells.

(4) Transplant more mature cells instead of naive ones. It has been observed that teratomas formed from iPSCderived NS/PCs were much smaller than those directly from iPSCs, indicating that predifferentiation of iPSCs can reduce certain aspects of tumorigenicity [43]. Consequently, grafting iPSCs directly in the treatment of SCI is not recommended. 
Taken together, these ways to address undifferentiated cell contamination in iPSC-derived NS/PC transplants are, at least in part, currently effective, but it seems impossible for some of these methods to be translated into clinical application due to either the invasive operation or time-consumed culture to weaken epigenetic memory. And we had better transplanted relatively mature iPSC-derived NS/PCs instead of iPSC itself.

4.2. Strategies to Prevent True Tumors. As for substantial tumors, we also have several effective steps to reduce the risk including the following:

(1) Change the reprogramming methods into integrationfree methods. Virally induced iPSCs with genomic integrations of transcriptional factors easily cause insertional mutagenesis and result in continual expression of residual factors in iPSCs [44]. Thus, instead of using integrative vectors like retrovirus or lentivirus, we need to pursue integration-free methods, not perturbing the genome. Episomal vector and Sender virus vector were once thought to be ideal nonintegrating methods, as the former works as extrachromosomal DNA in the nucleus while the latter is a method of transgene-free induction. But as the potential spontaneous integration by episomal vector and the involvement viral particles, both are limited to clinical applications. Subsequently, Woltjen et al. discovered that piggyBac transposons could be integrated into genomes of the host so the reprogramming factors that they carried were able to express continuously and stably [45]. Furthermore, the piggyBac transposons could be cut out of the genomes completely [45]. Afterwards, the advent of DNA-free and viral-free methods like recombinant proteins, messager RNA, and mature microRNA made iPSCs stride towards clinical use despite being technically challenging or inefficient. Of note, iPSCs of the first clinical trial were generated by the nonintegrative method of reprogramming with recombinant proteins [46].

(2) Avoid using transgenic factors of oncogenesis. The Yamanaka factors are competent enough to induce tumorigenesis playing important roles in the development and maintenance of cancer. It appears quite necessary to reduce reprogramming factors in order to decrease the possibility of tumor formation and hasten the clinical use. Nakagawa et al. initiated a series of experiments to test whether fewer factors are capable enough of inducing the stem cell. It was found that exogenous c-Myc was not necessarily needed to generate iPSCs [31]. They then found that exogenous Oct- 4 together with KLF4 or SOX2 could produce iPSCs from NSC. Furthermore, they discovered that transcriptional factor Oct-4 alone is sufficient to acquire iPSCs [41]. Although the lowreprogramming efficiency of them limits their applications, their attempt provides us with new ideas.
(3) Reduce undesirable DNA methylation. Decreasing DNA methylation of tumor suppressor genes and increasing that of oncogenes can certainly reduce the rate of tumor formation from iPSCs. The application of knocking down the maintenance methyltransferase DNMT1 or the demethylating agent like 5-AZA can reduce residual methylation of resulting cells and convert them to authentic pluripotent cells [33]. Besides, Mikkelsen et al. found that demethylation appears passage dependent [47]. Some reports showed that DNA methylation could be gradually erased as the cells were passaged $[33,39]$. Iida et al. [26], however, found that DNA methylation patterns became more unstable with cells passaged. Maybe, this can be accounted for the fact that the cell clones that they used were different indicating that the ability of passaging to gradually diminish methylation cannot be applicable to all clones.

(4) Establish reliable ways to distinguish the safe and unsafe cell clones. By virtue of the teratomaforming activity of the iPSC derivatives after their transplantation [28], we are capable of differentiating the safe iPSC clones from all cultured cell clones. Preevaluated safe clones can show significant therapeutic effects without tumor formation [16-18], while preevaluated unsafe clones demonstrate high rates of tumor formation. Iida discovered that methylation states of CAT and PSMD5 genes can be applied to discriminate between safe and unsafe hiPSC-NS/ PCs [26].

In brief, across the entire process of iPSC generation and NS/PC differentiation, there are steps that can be taken to reduce nonteratoma tumor formation. These strategies mentioned above just provide some possible way to circumvent the tumorigenicity, but I am afraid that there is still a long way from clinical applications.

\section{Conclusions}

Despite numerous therapeutic discoveries in the laboratory, to our knowledge, faithfully effective treatment for spinal cord injury remains unavailable. iPSC transplantation for SCI is currently an unrealistic strategy, but we have already recognized the huge potential of iPSCs for SCI because of their ability to self-renew and differentiate into various types of neural cells. In addition, iPSCs also avoid the ethical issues associated with some transplant sources and importantly can be performed in an autologous manner removing the need for immune suppression.

However, although the Takahashi group claimed that they were warranted to restart their clinical trials on iPSCs, safety concerns, especially tumorigenicity, still seriously limit considerations for clinical application, at least on SCI [48]. They once carried out the first clinical application of iPSCs in 2014, but were required to halt for some reasons. In this review, we focused on the two different routes of tumorigenicity and underlying mechanisms behind them. We also 
put forward some potential solutions to tumorigenesis. But in the current state, not enough is understood about underlying causes of tumor genesis from iPSC derivatives to completely elucidate the issue. More explorations and attempts need to be done in the future.

\section{Abbreviations}

ESCs: Embryonic stem cells

hiPSCs: Human iPSCs

iPSCs: Induced pluripotent stem cells

NS/PC: Neural stem cell or progenitor cell

SCI: Spinal cord injury.

\section{Conflicts of Interest}

The authors declare that they have no competing interests.

\section{Authors' Contributions}

Junhao Deng wrote the initial manuscript. Yiling Zhang, Yong Xie, and Licheng Zhang participated in drafting the manuscript. Peifu Tang revised the manuscript. All authors read and approved the final manuscript.

\section{Acknowledgments}

The authors thank Xie $\mathrm{Wu}$ and their laboratory members for their dedicated work. They are supported by the projects of the international cooperation and exchanges of the National Natural Science Foundation of China (81520108017).

\section{References}

[1] A. Singh, L. Tetreault, S. Kalsi-Ryan, A. Nouri, and M. G. Fehlings, "Global prevalence and incidence of traumatic spinal cord injury," Clinical Epidemiology, vol. 6, pp. 309-331, 2014.

[2] World Health Organization, "International perspectives on SCI," November 2013, http://www.who.int/mediacentre/fact sheets/fs384/en/.

[3] M. B. Bracken, M. J. Shepard, W. F. Collins et al., "A randomized, controlled trial of methylprednisolone or naloxone in the treatment of acute spinal-cord injury - results of the Second National Acute Spinal Cord Injury Study," The New England Journal of Medicine, vol. 322, no. 20, pp. 1405-1411, 1990.

[4] T. Qian, D. Campagnolo, and S. Kirshblum, "High-dose methylprednisolone may do more harm for spinal cord injury," Medical Hypotheses, vol. 55, no. 5, pp. 452-453, 2000.

[5] T. Qian, X. Guo, A. D. Levi, S. Vanni, and R. T. Shebert, "Highdose methylprednisolone may cause myopathy in acute spinal cord injury patients," Spinal Cord, vol. 43, no. 4, pp. 199-203, 2005.

[6] G. W. J. Hawryluk, J. Rowland, B. K. Kwon, and M. G. Fehlings, "Protection and repair of the injured spinal cord: a review of completed, ongoing, and planned clinical trials for acute spinal cord injury. A review," Neurosurgical Focus, vol. 25, no. 5, p. E14, 2008.

[7] B. A. Sabel, M. D. Slavin, and D. G. Stein, "GM1 ganglioside treatment facilitates behavioral recovery from bilateral brain damage," Science, vol. 225, no. 4659, pp. 340-342, 1984.
[8] T. Hashimoto and N. Fukuda, "Effect of thyrotropin-releasing hormone on the neurologic impairment in rats with spinal cord injury: treatment starting $24 \mathrm{~h}$ and 7 days after injury," European Journal of Pharmacology, vol. 203, no. 1, pp. 2532, 1991.

[9] A. J. Mothe and C. H. Tator, "Review of transplantation of neural stem/progenitor cells for spinal cord injury," International Journal of Developmental Neuroscience, vol. 31, no. 7, pp. 701-713, 2013.

[10] S. Strauss, "Geron trial resumes, but standards for stem cell trials remain elusive," Nature Biotechnology, vol. 28, no. 10, pp. 989-990, 2010.

[11] G. Kumagai, Y. Okada, J. Yamane et al., "Roles of ES cellderived gliogenic neural stem/progenitor cells in functional recovery after spinal cord injury," PLoS One, vol. 4, no. 11, article e7706, 2009.

[12] M. Nakamura and H. Okano, "Cell transplantation therapies for spinal cord injury focusing on induced pluripotent stem cells," Cell Research, vol. 23, no. 1, pp. 70-80, 2013.

[13] K. Takahashi and S. Yamanaka, "Induction of pluripotent stem cells from mouse embryonic and adult fibroblast cultures by defined factors," Cell, vol. 126, no. 4, pp. 663-676, 2006.

[14] I. H. Park, R. Zhao, J. A. West et al., "Reprogramming of human somatic cells to pluripotency with defined factors," Nature, vol. 451, no. 7175, pp. 141-146, 2008.

[15] K. Hochedlinger and R. Jaenisch, "Nuclear reprogramming and pluripotency," Nature, vol. 441, no. 7097, pp. 1061-1067, 2006.

[16] O. Tsuji, K. Miura, Y. Okada et al., "Therapeutic potential of appropriately evaluated safe-induced pluripotent stem cells for spinal cord injury," Proceedings of the National Academy of Sciences of the United States of America, vol. 107, no. 28, pp. 12704-12709, 2010.

[17] S. Nori, Y. Okada, A. Yasuda et al., "Grafted human-induced pluripotent stem-cell-derived neurospheres promote motor functional recovery after spinal cord injury in mice," Proceedings of the National Academy of Sciences of the United States of America, vol. 108, no. 40, pp. 16825-16830, 2011.

[18] Y. Kobayashi, Y. Okada, G. Itakura et al., "Pre-evaluated safe human iPSC-derived neural stem cells promote functional recovery after spinal cord injury in common marmoset without tumorigenicity," PLoS One, vol. 7, no. 12, article e52787, 2012.

[19] P. Lu, G. Woodruff, Y. Wang et al., "Long-distance axonal growth from human induced pluripotent stem cells after spinal cord injury," Neuron, vol. 83, no. 4, pp. 789-796, 2014.

[20] Y. Fujimoto, M. Abematsu, A. Falk et al., "Treatment of a mouse model of spinal cord injury by transplantation of human induced pluripotent stem cell-derived long-term selfrenewing neuroepithelial-like stem cells," Stem Cells, vol. 30, no. 6, pp. 1163-1173, 2012.

[21] T. Okubo, A. Iwanami, J. Kohyama et al., "Pretreatment with a $\gamma$-secretase inhibitor prevents tumor-like overgrowth in human iPSC-derived transplants for spinal cord injury," Stem Cell Reports, vol. 7, no. 4, pp. 649-663, 2016.

[22] Y. Pomeshchik, K. A. Puttonen, I. Kidin et al., "Transplanted human induced pluripotent stem cell-derived neural progenitor cells do not promote functional recovery of pharmacologically immunosuppressed mice with contusion spinal cord injury," Cell Transplantation, vol. 24, no. 9, pp. 1799-1812, 2015. 
[23] M. Ozaki, A. Iwanami, N. Nagoshi et al., "Evaluation of the immunogenicity of human iPS cell-derived neural stem/ progenitor cells in vitro," Stem Cell Research, vol. 19, pp. 128$138,2017$.

[24] S. Nishimura, A. Yasuda, H. Iwai et al., "Time-dependent changes in the microenvironment of injured spinal cord affects the therapeutic potential of neural stem cell transplantation for spinal cord injury," Molecular Brain, vol. 6, no. 1, p. 3, 2013.

[25] S. E. Nutt, E. A. Chang, S. T. Suhr et al., "Caudalized human iPSC-derived neural progenitor cells produce neurons and glia but fail to restore function in an early chronic spinal cord injury model," Experimental Neurology, vol. 248, pp. 491503, 2013.

[26] T. Iida, A. Iwanami, T. Sanosaka et al., "Whole-genome DNA methylation analyses revealed epigenetic instability in tumorigenic human iPS cell-derived neural stem/progenitor cells," Stem Cells, vol. 35, no. 5, pp. 1316-1327, 2017.

[27] G. Itakura, S. Kawabata, M. Ando et al., "Fail-Safe system against potential tumorigenicity after transplantation of iPSC derivatives," Stem Cell Reports, vol. 8, no. 3, pp. 673-684, 2017.

[28] K. Miura, Y. Okada, T. Aoi et al., "Variation in the safety of induced pluripotent stem cell lines," Nature Biotechnology, vol. 27, no. 8, pp. 743-745, 2009.

[29] C. J. Parr, S. Katayama, K. Miki et al., "MicroRNA-302 switch to identify and eliminate undifferentiated human pluripotent stem cells," Scientific Reports, vol. 6, no. 1, article 32532, 2016.

[30] M. R. Simpson-Abelson, J. L. Loyall, H. K. Lehman et al., "Human ovarian tumor ascites fluids rapidly and reversibly inhibit $\mathrm{T}$ cell receptor-induced NF- $\mathrm{\kappa B}$ and NFAT signaling in tumor-associated T cells," Cancer Immunology Research, vol. 13, no. 3, p. 14, 2013.

[31] M. Nakagawa, M. Koyanagi, K. Tanabe et al., "Generation of induced pluripotent stem cells without Myc from mouse and human fibroblasts," Nature Biotechnology, vol. 26, no. 1, pp. 101-106, 2008.

[32] R. Lister, M. Pelizzola, Y. S. Kida et al., "Hotspots of aberrant epigenomic reprogramming in human induced pluripotent stem cells," Nature, vol. 471, no. 7336, pp. 68-73, 2011.

[33] K. Kim, A. Doi, B. Wen et al., "Epigenetic memory in induced pluripotent stem cells," Nature, vol. 467 , no. 7313 , pp. $285-$ 290, 2010

[34] S. Nori, Y. Okada, S. Nishimura et al., "Long-term safety issues of iPSC-based cell therapy in a spinal cord injury model: oncogenic transformation with epithelial-mesenchymal transition," Stem Cell Reports, vol. 4, no. 3, pp. 360-373, 2015.

[35] C. E. Goldring, P. A. Duffy, N. Benvenisty et al., "Assessing the safety of stem cell therapeutics," Cell Stem Cell, vol. 8, no. 6, pp. 618-628, 2011.

[36] U. Ben-David and N. Benvenisty, "The tumorigenicity of human embryonic and induced pluripotent stem cells," Nature Reviews Cancer, vol. 11, no. 4, pp. 268-277, 2011.

[37] L. Warren, P. D. Manos, T. Ahfeldt et al., "Highly efficient reprogramming to pluripotency and directed differentiation of human cells with synthetic modified mRNA," Cell Stem Cell, vol. 7, no. 5, pp. 618-630, 2010.

[38] S. C. Mack, C. G. Hubert, T. E. Miller, M. D. Taylor, and J. N. Rich, "An epigenetic gateway to brain tumor cell identity," Nature Neuroscience, vol. 19, no. 1, pp. 10-19, 2015.

[39] J. M. Polo, S. Liu, M. E. Figueroa et al., "Cell type of origin influences the molecular and functional properties of mouse induced pluripotent stem cells," Nature Biotechnology, vol. 28 , no. 8, pp. 848-855, 2010.

[40] J. B. Kim, H. Zaehres, M. J. Araúzo-Bravo, and H. R. Schöler, "Generation of induced pluripotent stem cells from neural stem cells," Nature Protocols, vol. 4, no. 10, pp. 1464-1470, 2009.

[41] J. B. Kim, V. Sebastiano, G. Wu et al., "Oct4-induced pluripotency in adult neural stem cells," Cell, vol. 136, no. 3, pp. 411419, 2009.

[42] A. Bedel, F. Beliveau, I. Lamrissi-Garcia et al., "Preventing pluripotent cell teratoma in regenerative medicine applied to hematology disorders," Stem Cells Translational Medicine, vol. 6, no. 2, pp. 382-393, 2017.

[43] Z. Liu, Y. Tang, S. Lu et al., "The tumourigenicity of iPS cells and their differentiated derivates," Journal of Cellular and Molecular Medicine, vol. 17, no. 6, pp. 782-791, 2013.

[44] X. Kang, Q. Yu, Y. Huang et al., "Effects of integrating and non-integrating reprogramming methods on copy number variation and genomic stability of human induced pluripotent stem cells," PLoS One, vol. 10, no. 7, article e131128, p. e0131128, 2015

[45] K. Woltjen, I. P. Michael, P. Mohseni et al., "piggyBac transposition reprograms fibroblasts to induced pluripotent stem cells," Nature, vol. 458, no. 7239, pp. 766-770, 2009.

[46] M. Takahashi, "Retinal cell therapy using iPS cells," Rinsho Shinkeigaku, vol. 53, no. 11, article 1016, 2013.

[47] T. S. Mikkelsen, J. Hanna, X. Zhang et al., "Dissecting direct reprogramming through integrative genomic analysis," Nature, vol. 454, no. 7200, pp. 49-55, 2008.

[48] M. Mandai, A. Watanabe, Y. Kurimoto et al., "Autologous induced stem-cell-derived retinal cells for macular degeneration," New England Journal of Medicine, vol. 376, no. 11, pp. 1038-1046, 2017. 


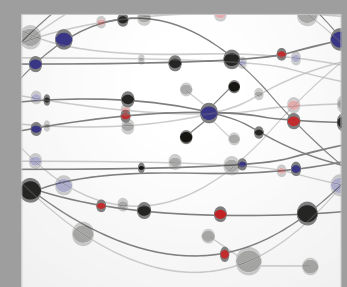

The Scientific World Journal
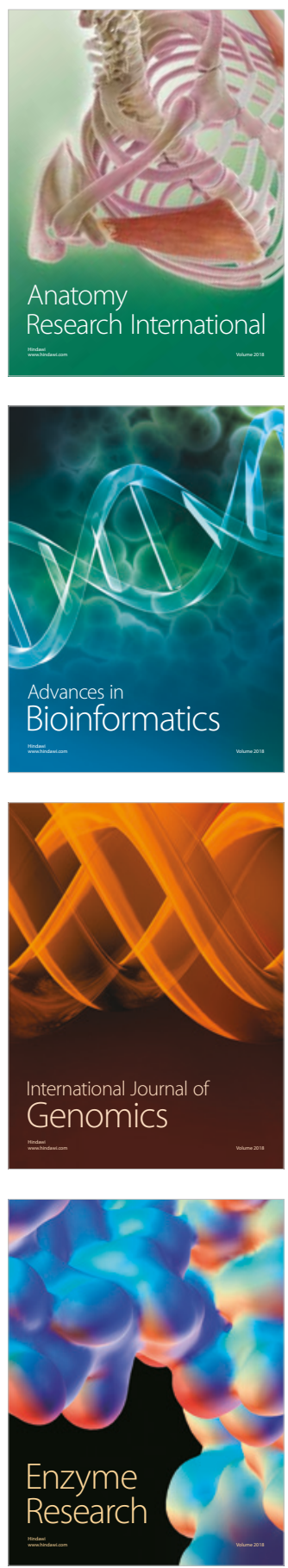
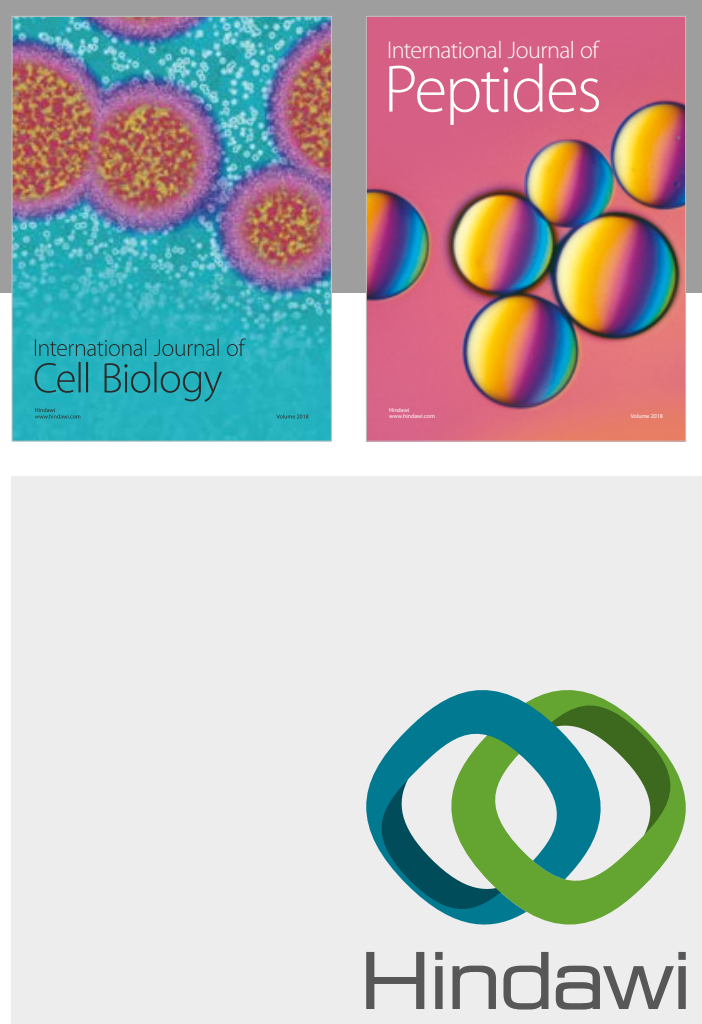

Submit your manuscripts at

www.hindawi.com
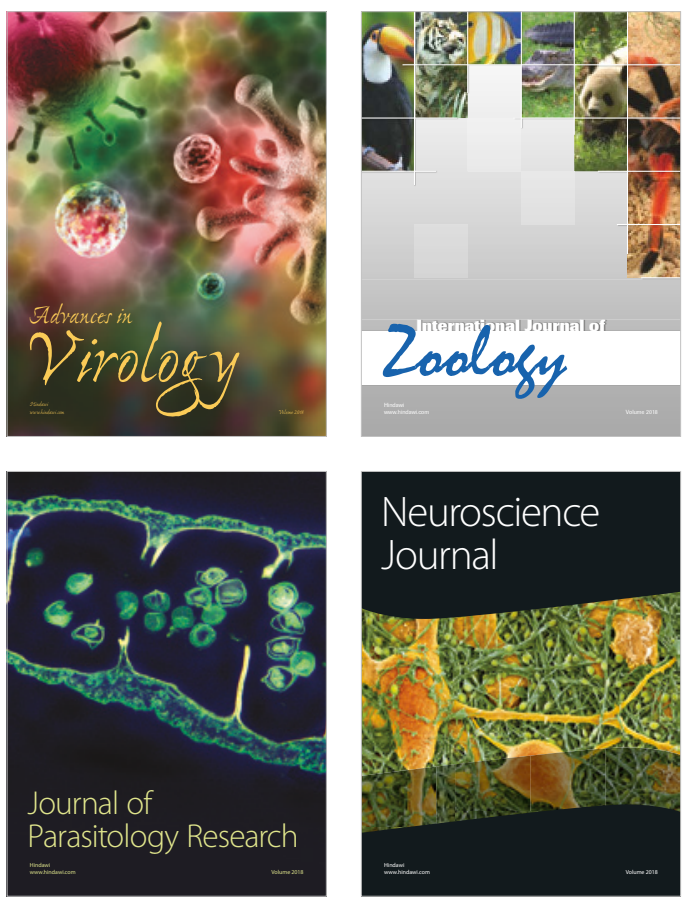
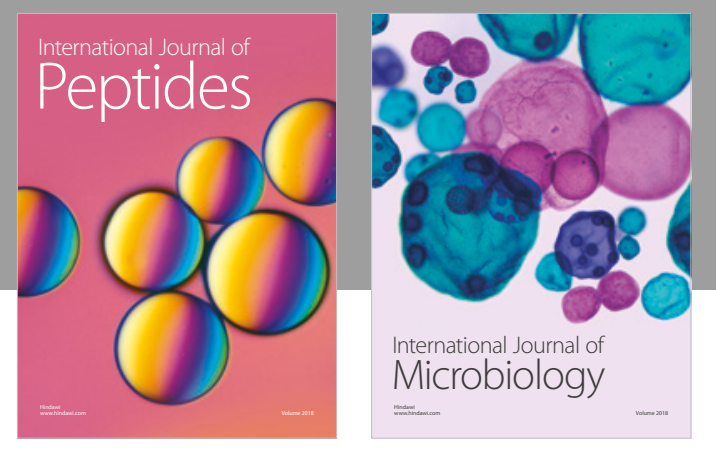

nternational Journal of Microbiology
Journal of
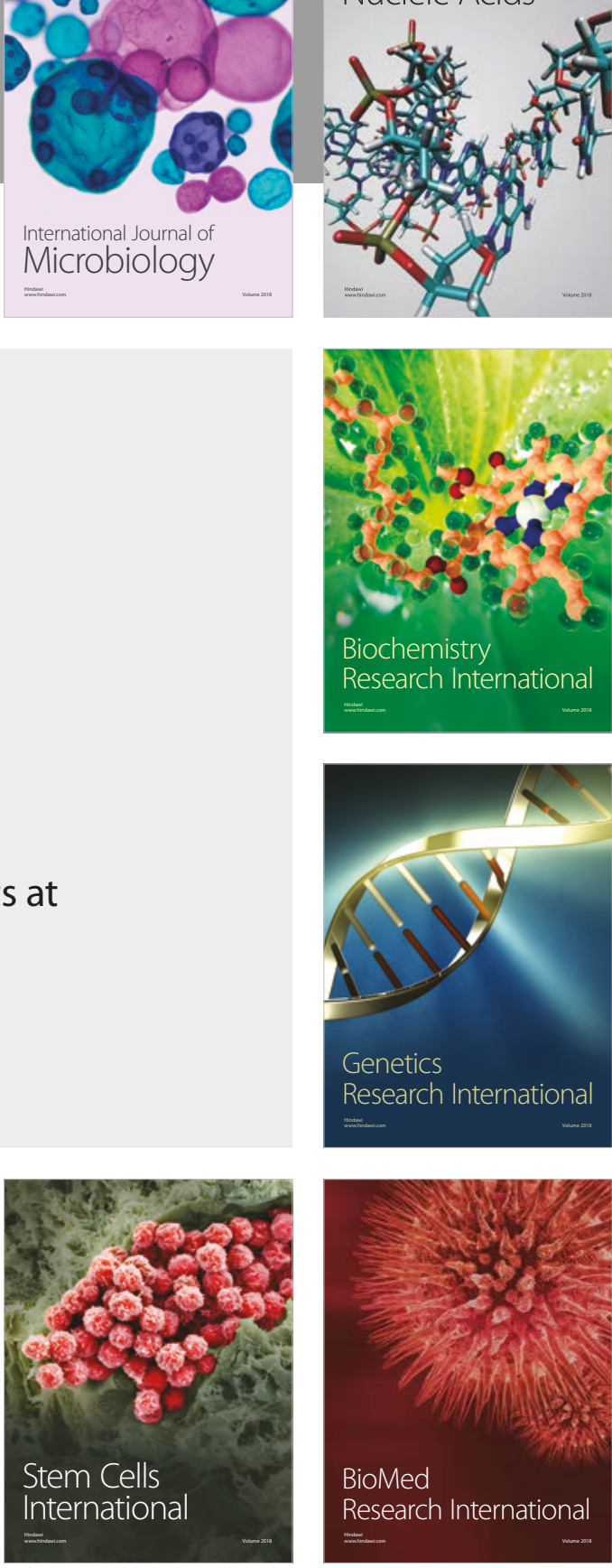
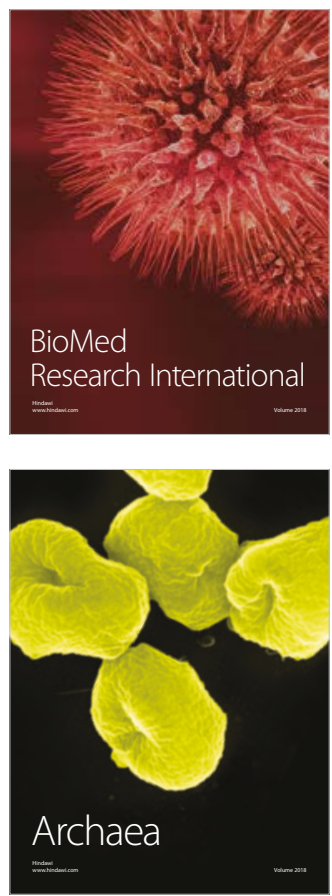